\title{
Téoros
}

Revue de recherche en tourisme

\section{Destination : Bas-Saint-Laurent}

Le déploiement de la villégiature à la confluence Saguenay Saint-Laurent

\section{Serge Gagnon}

Volume 20, numéro 1, printemps 2001

Mers et littoraux

URI : https://id.erudit.org/iderudit/1071908ar

DOI : https://doi.org/10.7202/1071908ar

Aller au sommaire du numéro

\section{Éditeur(s)}

Université du Québec à Montréal

\section{ISSN}

0712-8657 (imprimé)

1923-2705 (numérique)

Découvrir la revue

Citer cet article

Gagnon, S. (2001). Destination : Bas-Saint-Laurent : le déploiement de la villégiature à la confluence Saguenay - Saint-Laurent. Téoros, 20(1), 28-33. https://doi.org/10.7202/1071908ar d'utilisation que vous pouvez consulter en ligne.

https://apropos.erudit.org/fr/usagers/politique-dutilisation/ 


\section{Destination : Bas-Saint-Laurent

\author{
Le déploiement de la villégiature \\ à la confluence Saguenay - Saint-Laurent
}

\section{Serge Gagnon}

Cet article se propose de relativiser l'interprétation classique de la transformation géohistorique et de lorganisation touristique de la confuence Saguenay-Saint-Laurent'. Il s'agit de montrer que le déploiement d'une villégianure somptuaire dans cette région, au cours du XIX siècle, représente l'accomplissement de progranmes d'appropriation. Des motivations autres que socio-économiques ont contribué à la mise en place de cette aire touristique, ainsi qu'à sa va= lorisation.
D ans le sillage d'un mouvement de sa lubrité urbaine, divers acteurs convoitent les rivages du Saint-Laurent dès le début du XIX siècle. Une lutte s'engage entre eux, la partie se jouant sur le terrain de l'appropriation territoriale des positions bas-laurentiennes. Dans cette optique, l'activité économique du tourisme est venue rentabiliser une occupation ayant présupposé l'appropriation géopolitique d'un espace qui était dejà investi de valeurs culturelles liées au courant romantique qui, à l'époque, déferlait sur l'Amérique du Nord.

Le phénomene touristique, plus précisément la villégiature au Québec, est apparu à la fin du XVII ${ }^{e}$ siècle, principalement dans la région du Bas-Saint-Laurent (Beaudet et Lamothe, 1996 ; Blanchard, 1960 ; Brière, 1967 ; Dubé, 1986; Gagnon, F., 1992 ; Gagnon, S., 1996 ; St-Amour, 1979 ; Samson, M., 1987 et 1988 ; Stafford et Samson, 1986). Les stations touristiques du BasSaint-Laurent, autour de 1850 , étaient très populaires ; en effet, Roger Brière (cité par F. Gagnon, 1992 : 102) dit de la région qu'elle est \& [...] ']a' zone touristique par excellence de l'Est de l'Amérique du Nord britannique $*$. Selon une étude exhaustive de France Gagnon sur le développement du tourisme au $\mathrm{XIX}^{e}$ siècle versus les moyens de transport (route, vapeur et chemin de fer), 4. Cette région [le Bas-Saint-Laurent] existait en tant que ierritoire touristique, dotée de stations en puissance et d'une clientèle réelle et potentielle certaines ; la navigation à vapeur est venue stimuler considérablement un développement déjä amorcé w (idem : 104).

Les nombreuses stations de villégiature de Cacouna, de Kamouraska, de Métis-sur-
Mer, de Murray Bay, de Notre-Dame-duPortage et de Tadoussac - que l'on désigne globalement du nom de Lower St. Lawrence dans les guides touristiques du $\mathrm{XIX}^{e}$ siecle - incarnent la saisie de la a nature comme valeur attractive. Les littoraux et les massifs montagneux sont ainsi mis en valeur par les croisières sur les fameux * bateaux blancs $*$ (Normand, 1997 ; Tittley, 1995 ; Lessard, 1993).

\section{Kamouraska: le lieu de rendez-vous de l'élite canadienne-française}

Déjà lors de sa tournée d'inspection en 1813, Joseph Bouchette identifie Kamouraska comme étant un centre de villégiature.

Le village de Kamouraska se trouve dans un site enchanteur, pres du chemin principal [...]. On y voit des familles tout à fait respectables, ainsi que quelques gros marchands ei des artisans ; wn ou deux hôtels y donnent un bon service aux voyagears. Durant l'êtê, de nombreux touristes y sejoument pour refaire leur sante, l'endroit ayant la réputation d'être un des plus sains de la province. C'est aussi une place d'eau où beaucoup de gens viennent prendre des bains de mer. Le manoir, résidence de $M$. Tache, se trowve en un endroit de choix, près dufleuve, à une courte distance du village (Paradis, 1948 : 168).

Roger Brière désigne Kamouraska comme le lieu de rencontre de l'élite canadiennefrançaise. 


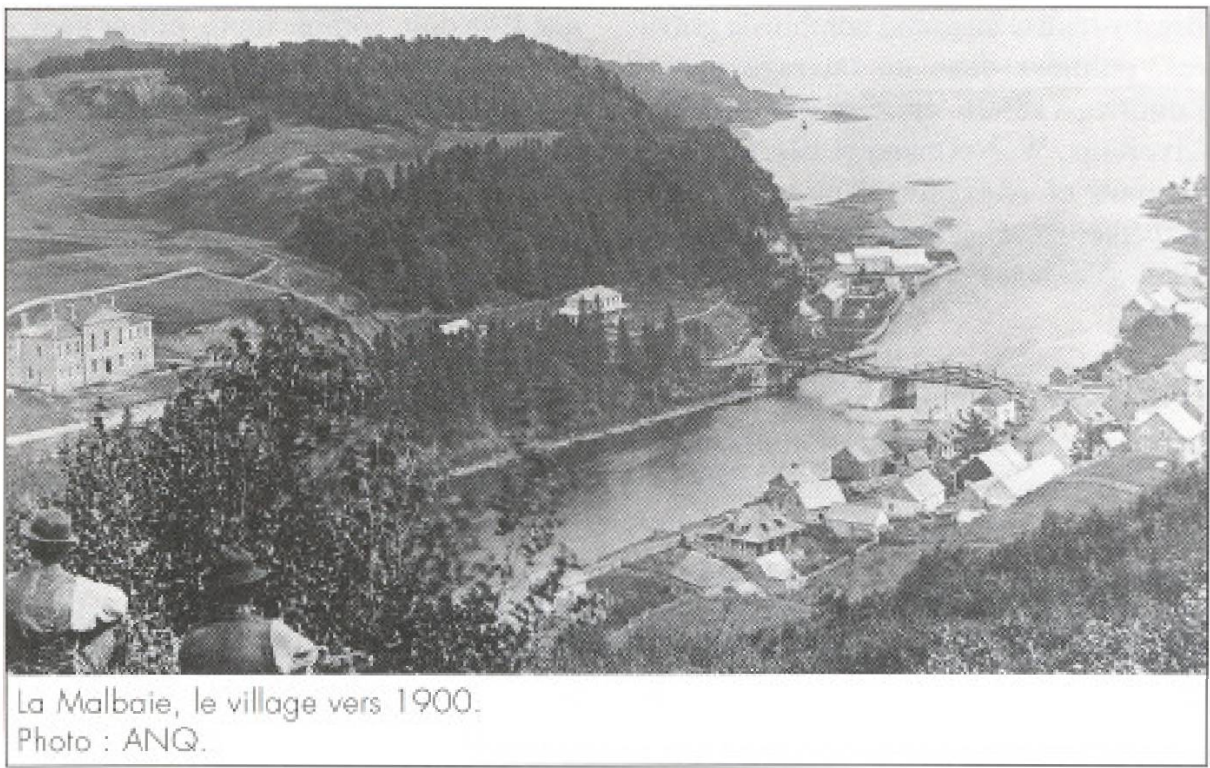

Le premier séjour d'été auquel se domèrent rendez-vous les familles canadiennes-françaises jouissant de quelque fortune, fut Kamouraska. Ce village est le seul rendez-vous estival dont nous ayons trouvé mention avant l'áge des bateaux à vapeur et du chemin de fer [... J. Nulle part cependant, les villégiateurs n'étaient en nombre suffisant pour qu'on pât parler de station de villégiature au sens actuel. Kamouraska semble avoir été la seule exception, dès les premieres années du siècle. Ce village fut en effet le premier du Québec à recevoir des citadins en vacances. Sa célébrité datait de l'époque des seigneurs Taché et des fêtes resplendissantes qu'il y donnair $(1967: 86$ 88).

Au début du XIX ${ }^{\mathrm{r}}$ siècle, Kamouraska possède tous les attributs d'une station de villégiature. Un mouvement d"évasion, du centre du village d'origine vers la périphérie en bordure du fleuve, permet d'y organiser les premières activités de loisir.

Ce déplacement géographique [...] semble avoir eu des effets bénéfiques. Le village a pris de l'ampleur et, selon Joseph Bouchette, arpenteur-general, était devenu en 1813 un lieu de villégiature recherché. Kamoreraska a vu sa population culminer à près de 6000 habitants en
1827. En 1849, La localité est devenue un chef-lieu régional en accueillant une cour supérieure de justice (Larocque, 1993: 52).

Plusieurs auteurs dépeignent la région ; par exemple, Alexandre Paradis décrit ainsi l'aménagement du secteur près du fleuve : * Tout près de là habite le seigneur, là sont les magasins, les hôtels, les quais, les hommes de profession et les touristes (1948: 180 ; et Martin, J., 1998). PaulLouis Martin, pour sá part, décrit le site de villégiature: « [le front] de mer accidenté et les terrasses rocheuses vont alors ces d'été a l'architecture très caractérisée \% (1996 : 2). Philippe Dubé fait lạ description des équipements typiques d'une station de bain de mer à l'époque : * Au XIX" siècle, les stations de bain de mer doivent obligatoirement comprendre une ou plusieurs auberges, des villas à louer, une église catholique, un temple protestant, un grand hôtel et une vue sur la mer $n(1983: 220)$.

Kamouraska est donc la première authentique station touristique du Canada. Au début đu XIX" siècle, le seigneur du lieu, Pascal Taché, qui avait siégé pendant deux ans à la Chambre d'Assemblée du BasCanada (1798-1800), se préoccupe particulièrement de sa seigneurie où il accueille l'élite du temps (Laberge, 1993: 162). L'endroit est surtout fréquenté par les Canadiens français de haut rang. L'élite se peupler de villas, d' hôtels et de résiden-
* aristocratique ss'y réunit pour discuter de politique et de stratégie. En ce début de siècle, cette élite sent cependant venir le vent de la bourgeoisie et doit trouver de nouveaux défis pour mobiliser la population canadienne-française. Jean-Pierre Saint-Amour note, à l'égard de la clientèle qui fréquente la station, que « La vallée du Saint-Laurent a connu ses premiers établissements de sejour estival avec l'émergence d'une haute société̉ et d'une élite. Ce fut d'abord à Kamouraska que se rencontrèrent les familles aisées canadiennes-françaises du début du dix-neuvième siècle. Il semble que ce fut un des seuls centres fréquentés par les francophones \% (1979: 19). De son côté, Brière rapporte les propos d'Arthur Buies et de James MacPherson Le Moine, grands chroniqueurs et voyageurs de la fin du XIX siècle.

On s'y rend de Montréal et de Québec bien avant que des places d'eau comme Pointeau-Pic et Cacouna ne soient connues. Buies peut ainsi dire que « les autres endroits ne comptaient pas * et que le manoir des seigneurs Taché « avait reçu pendant un quart de siècle tout ce que le pays renfermait d'hommes éminents dans la vie publique ou distingués par la naissance et la position \%. Pour MacPherson Le Moine, $\approx$ before the area of steamers, in fact even as late as 1850 , Kamouraska was the Brighton of Lower Canada $*(1967 ; 88)$.

Contrôlé par les acteurs canadiens-français, le site de villëgiature de Kamouraska semble avoir été l'endroit où l'on a établi le plan de l'appropriation du Saguenay aux fins de la colonisation. Il est tentant de faire le parallèle entre un Kamouraska, première station touristique fréquentée par l'élite aristocratique canadienne-française, et un autré Kamouraska, site stratégique des tentatives de conquête du territoire saguenayen ou jeannois. Le fait que la notoriété de cet endroit fashionable ait périclité, à partir de 1870 , signifierait alors que les acteurs aristocrates ont perdu la bataille du Saguenay au profit des acteurs bourgeois (Gagnon, S., 1996: 79-103).

Brière décrit ainsi l'abandon de Kamouraska : « Malgré la concurrence des sta- 
tions où se déploya la mode anglaise à partir de 1850-1860, Kamouraska ne périclita que très lentement [...]. De nouvelles stations devaient acquérir une renommée dans la seconde moitié du XIX ${ }^{e}$ siècle, en particulier Pointe-au-Pic, Cacouna et Métis \$ (1967: 88). Dans le même ordte d'idées, Paradis note que la popularité de Kamouraska diminue à partir des années 1850-1860.

Il ne faut pas oublier les touristes attirés à Kamouraska par ses paysages sereins, son climat salubre, son atmosphère saline, ses bains de mer. Déjà en 1813, note Bouchette, ils viennent nombreux y passer la belle saison. Jusque vers 1855 , c'est pratiquement l'unique plage à la mode, «Aller à l'eau salée, écrit Buies, veut dire aller à Kamouraska $\%$. Les familles les plus à l'aise et les plus distinguées de Québec et d'ailleurs s'y rencontrent. Selon Buies, vers 1873 des enthousiastes projettent de construire un hôtel sur l'une des îles en face et d'y aménager un quai pour permeture au *steamboat" de faire descendre les villégiateurs. Mais après 1860 , avec la construction de l'Intercolonial jusqu'au Bic et l'êtablissement d'une ligne maritime qui se rend à la Malbaie, Saint-Irénée et Tadoussac, Kamouraska subit une forte concurrence et voit un grand nombre de ses touristes déserter vers les nouveaux centres $(1948: 185)$.

Bref, au cours de la première moitié du $\mathrm{XIX}^{\circ}$ siècle, Kamouraska est un rendezvous estival de l'aristocratie canadiennefrançaise, aristocratie qui fléchit devant l'émergence de la nouvelle bourgeoisie canadienne.

\section{Le front romantique de Charlevoix : I'émergence de la bourgeoisie canadienne}

Au Québec, les artistes ont contribué à diffuser les valeurs associées à certaines formes sensibles de la vallée laurentienne et des massifs qui l'enserrent. L'exemple de Charlevoix est remarquable à cet égard (Gagnon, S., 1998 et 1996 ; Dube, 1983 , 1985, 1986 et 1995) : George Heriot, de passage à Murray Bay à la toute fin du XVIII' siècle, en est d'ailleurs un précurseur. Au milieu du XIX"e siècle, John J.
Bigsby réalise un grand nombre de gravures, publiées dans un ouvrage paru à Londres (1850) et d'autres, comme Livernois, W. Notmann et fils, Clarence Gagnon et Alexander Young Jackson, perpétueront cette tradition (Des Gagniers, 1994 ; Vézina, 1977 ; Villeneuve, 1996 et 1999).

Un massif de villégiature fashionable est mis en place à Murray Bay (Pointe-au-Pic) en 1853 (Gagnon, S., 1996 et 1998), La colonisation du Saguenay en est la condition d'appropriation et de valorisation bourgeoises. L'aristocratie seigneuriale occupe déjà la position convoitée par l'acteur bourgeois et elle contrôle la mobilité des populations locales; 1 'espace ciblé par la bourgeoisie n'est donc pas immédiatement disponible.

En organisant la colonisation du Saguenay, la bourgeoisie parvient ainsi à saper le pouvoir de l'acteur seigneurial. Elle dévalue temporairement les positions convoitées sur le littoral, en offrant une avenue de remplacement aux censitaires dont la mobilité était jusque-là contrôlée par le pouvoir d'Ancien Régime et ce, grâce au verrouillage de l'arrière-pays du Saguenay décrété à Londres.

La dévalorisation des fiefs littoraux facilite la formation d'une réserve bourgeoise. $L$ 'investissement de valeurs romantiques dans ces domaines devenus disponibles détermine une revalorisation foncière, laquelle stimule l'édification des formes architecturales qui mettent le site en valeur. En effet, une étude menée par Raymond Malo au début des années 1980, montre que « les manoirs de villégiature de Pointe-au-Pic ont évacué des aires agricoles au tournant de ce siècle, et que la spéculation fonciêre y a sévi dès la seconde moitié du XIX" siècle [...] dans un coin alors perdu, non relié aux villes, ni par la route, ni par chemin de fer $\leadsto$ (Ritchot, $1981: 57)$

Selon Dubé, cela signifie que Charlevoix constituerait « un pôle majeur de définition figurative du Canada où le territoire se travestit volontiers, pour les besoins de la cause, en un emblème national signifiant que l'on doit, somme toute, porter haut $\%$ (1996: 6-7). Dans cette optique, l'absence d'une liaison ferroviaire et routière pourrait avoir été davantage voulue que subie. $L$ 'isolement relatif de la sous-région de Murray Bay (Pointe-au-Pic) aurait participé d'une mise en réserve d'un territoire à valoriser, stimulant ainsi le désir d'une clientèle ayant les moyens d'être périodiquement oisive (Gagnon, S., 1996 et 1998).

Le cas de Charlevoix est exceptionnel, dans la mesure où il a confronté les acteurs aristocratiques et bourgeois d'emblée animés par une quête des mêmes valeurs. Il pourrait cependant ne pas avoir été unique. À cet égard, le Bas-Saint-Laurent a été une zone de rivalité entre des acteurs différents, relevant de la bourgeoisie canadienne.

\section{Le Bas-Saint-Laurent : au cour d'une " rivalité $"$ de $"$ rivages}

À partir du milieu du XIXe siècle, d'autres acteurs sociaux importants entrent en scène. Ce sont les compagnies de navigation et de chemin de fer (De Blois-Martin, 1997). Deux d'entre elles, la Compagnie du Richelieu ( $R$ \& O) et la St. Lawrence Steamboat Navigation Cie de la famille Molson (SLSC), se livrent une bataille pour le contrôle des positions de la région Lower St. Lawrence. Rappelons que, dans les guides touristiques au XIX siècle, les régions de Charlevoix et du Bas-SaintLaurent font partie de l'ensemble touristique ainsi nommé. Ce domaine comprend aussi le Bas-Saguenay et la Côte-du-Sud (Tadoussac, Pointe-au-Pic, Cacouna, Notre-Dame-du-Portage). C'est le vapeur Accommodation, propriété de John Molson, qui fait le premier voyage MontréalQuébec en 1809. Dès 1830, le vapeur Waterloo offre occasionnellement des départs pour La Malbaie à partir de Québec.

Bientôt, deux secteurs situés de part et d'autre du grand fleuve rivalisent de popularité, ceux de Charlevoix et du BasSaint-Laurent [...]. Les deux grandes régions de villégiature, dont Rivière-duLoup et La Malbaie sont en quelque sorte les charnières, accueillent une clientèle fort 
distinguée [...]. Mais les villages de la rive nord ne cèdent rien a leurs rivaux d'en face (Prévost, 2000: 31 et 35).

Un tếmoin privilégié de l'éppoque, James MacPherson Le Moine, met en garde la population de Cacouna contre la menace de la rive nord:

Bons habitants, voulez-vous m'en croire: Ne tuez pas la poule qui vous pond les aufs d'or $\$[\ldots]$. Velltez à ce qu'on hi laisse au moins les grosses plumes [les Molson]; autrement, elle s'envolera dans l'Tutercolonial, à Matane, à Gaspé même, ou par les steamers, à Tadoussac, à la Malbaie. N'en voulez pas à un vrai ami, qui a le courage de vous dire la vérité : Il faut tant de courage, ces années-ci, pour le dire (1872: 331$)$.

S'agit-il d'une rivalité entre les promoteurs de la navigation à vapeur et ceux du chemin de fer ? Certains commentateurs le pensent.

Il y a lieu de s'interroger, après le deuxieme boom ferroviaire des annees 1870 , sur le sort qui est maintenant réservé à la navigation à vapeur dans la faveur des touristes. Sur les grands circuits que la navigation à vapeur avait jadis rendu possibles, il y a maintenant des liaisons ferroviaires [...]. C'est probablement à cause de cette concurrence croissante du train, ajoutée a celle que lai live une autre compagnie de navigation (la * Union Line s) /St. Lawrence Steam Navigation Company de la fomille Molson], que la Compagnie du Richelieu instaure, des le débu des annees 1870 . un trajet de jour entre Montréal en Québec (Gagnon, F., 1992 : 131).

D'autres commentateurs, en revanche, doutent des répercussions de la compétition entre les moyens de transport par eau et par rail.

Présumée plas que démontré, la question de la rivalite rail-ean demeure pour tout dire un parent pau- we de notre histoire des transports. Au-delà des oppositions apparentes, le problème des relations entre les deux modes de transports devra un jour, croyons-nows, etre egalement posé da point de vue de la complé mentarité.

Normand, à qui l'on doit le passage prét= cédent (1997: 135), a probablement raison. En effet, le jumelage train-vapeur est très populaire dans la région du Bas-SaintLaurent, ailleurs aussi : dans les Cantonsde-l'Est, "La navigation à vapeur complète le rail tant pour le transport que pour la croisiere $n$ (Auger, 1993:60).

En 1860, le Grand Tronc atteint Rivière-du-Loup, offrant aux touristes la possibilite de réduire le temps de transport entre Montréal et Québec et les stations du Bas-SaintLaurent et du Saguenay; plusieurs optent en effet pour un trajet jume= lant le train et le vapeur, rejoignant seulemen à Riviere-du-Loup les vapeurs sur lesquels ils accomplissent le reste da trajet vers La Malbaie, Tadoussac ou le Saguenay. Par ces progrès dans les communications, la région, dejà éveillée à la vie touristique, recoit un puissant stimulant (Gagnon, F., $1992 ; 121$ ).

Alors, comment expliquer l'affrontement réel entre les deux entreprises de navigation R \& O et SLSC ? Comme le soutient Normand, il existe une complémentarité entre le rail et l'eau. La rivalité concernerait plutôt les acteurs qui contrôlaient les entreprises intếressées au développement de ces deux modes de transports. Les acteurs auraient rivalisé pour s'approprier les positions convoitées de la région du Bas-Saint-Laurent, et non pas pour favori= ser un moyen de transport aux dépens d'un autre.

Voyons maintenant comment la séquence des événements rend compte de la rivalité, non pas entre moyens de transport, mais entre acteurs. Dès 1853, au même moment où le gouvernement de Londres construit un quai sur pilotis à Pointe-au-Pic (Dubé, 1986), la compétition se fait sentir sur la liaison Montréal-Québec : \& Les competiteurs se multiplient. Molson trouve des compétiteurs particulièrement entreprenants dans la Torrance Line de David Torrance [...] mais également dans la Compagnie du Richelieu (Tittley, 1995 : 12). La Torrance Line sera absorbee par la R \& O en 1857.

En 1874, la grande bourgeoisie commence a fusionner les compagnies de navigation. Le rêve de contrôler la circulation maritime sur le Saint-Laurent pointe à l'horizon. Dans un premier temps, on assiste à la naissance de la Richelieu and Ontario Navigation Company ( $R$ \& O) qui permet d'offrir des croisières du Niagara jusqu'à Québec. Dans un article récent, Luc Tittley décrit l'ascension rapide de cette compagnie de navigation qui débute autour de 1860 :

Le nowvement de consolidation devait se poursuive avec des ententes de partage de marchés avec la

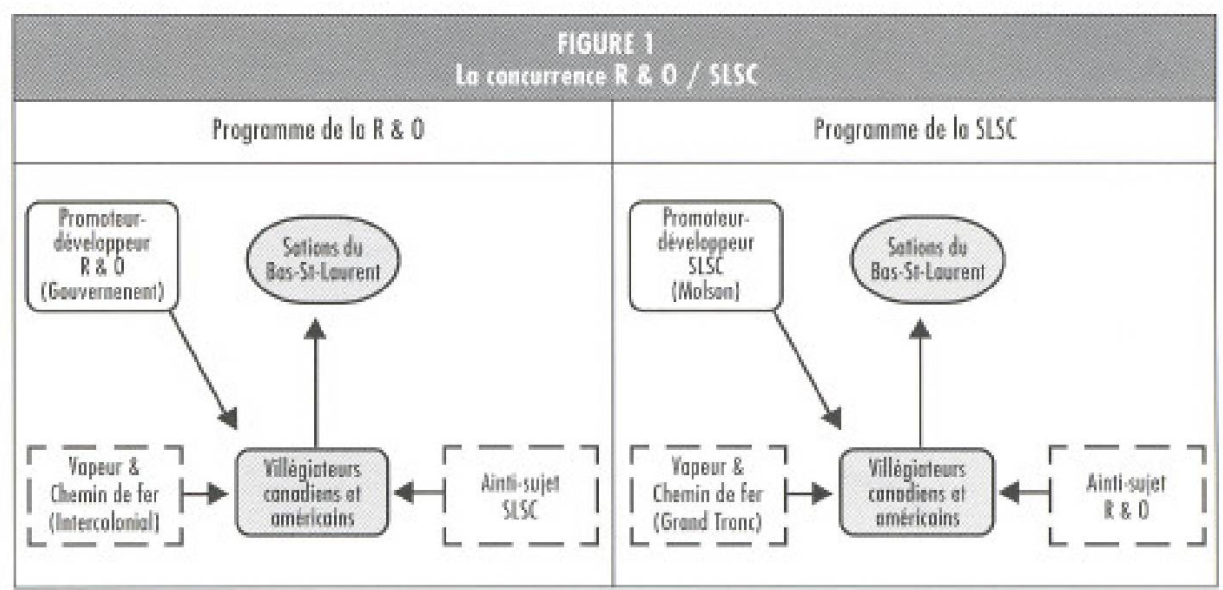


Canadian Steam Navigation Company de Sir Hugh Allan qui desservait le Saint-Laurent en amont de Montréal et le lac Ontario. Cette entreprise devait évenuellement s'intégrer à la Compagnie du Richelieu en 1874 et donner naissance à la Richelieu and Omario Navigation Company (Tittley, 1995: 12).

De son côté, la SLSC acquiert en 1875 lạ Quebec and Trois-Pistoles Steamboat Co., laquelle assurait le service sur le Saguenay avec le vapeur dénommé Saguenay. Comme la $R$ \& $O$ ne dessert pas cette route et s'arrête à Québec, « Les passagers de la Compagnie du Richelieu doivent, à cet egard, s'embarquer sur [1'Union] et d'autres (Saguenay, Clyde et St. Lawrence) pour faire l'excursion $x$ (Dubé, 1986: 72).

Le prestige qui auréole le tour du Saguenay ne fait qu'augmenter el, avec lui, celui des stations sises entre Québec et Chicoutimi. Sous la présidence d'Abraham Joseph, de Quc$b e c$, la St. Lawrence Steam Navigation Company remplace la Compagnie des Remorqueurs du SaintLaurent et offe un service de première classe a une clientèle de visileurs $[\ldots]$ (Idem : 76).

A la fin du XIX ${ }^{e}$ siềcle, les compagnies de navigation développent une stratégie qui vise l'appropriation des établissements

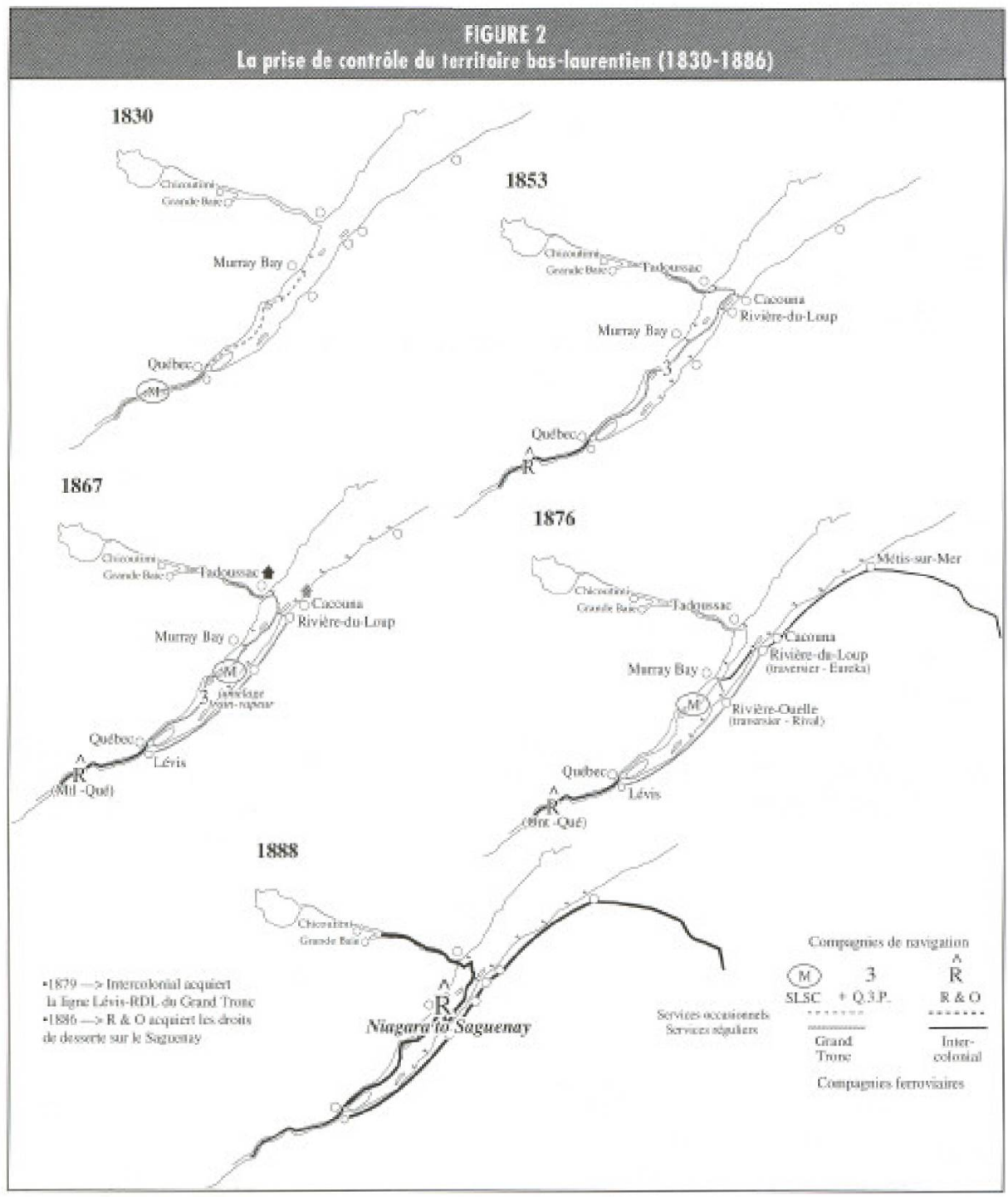

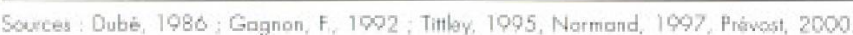

hôteliers que leurs navires de croisière, les * bateaux blancs *, fourniront en clients, ce qui fait dire à Claude Bergeron que : * Ce sont les compagnies de transport qui, pour mieux exploiter leur équipement et multiplier leurs profits, ont construit les grands hôtels de villégiature, comme au Canada le Canadien Pacifique et la Richelieu and Ontario Navigation Company, devenue par la suite la Canada Steamship Lines * (1989:62). La stratégie de la $\mathrm{R} \& \mathrm{O}$ est très claire.

Naturellement, pareille affluence ne peut laisser indifferentes les compagnies de transport. Comme on l'a vu, ces dernieres y trouvent l'occasion d'élargir la gamme de leurs services : elles ouvrent des hôtels là précisement où leurs vapeurs font escale, soit à Tadoussac et à La Malbaie. C'est ainsi que la Richelieu and Ontario Navigation Company prend possession de tout le terrain qui domine le quai de Pointeau-Pic, sur le haut de la falaise, où se trouvait justement la Chamard's Lorne House (Dubé, 1986: 92).

\section{Conclusion}

Grâce à l'acquisition des lignes du Saguenay en 1886 , la $R \&$ O contrôle, au tournant du XIX" siècle, la navigation de plaisance, des Grands Lacs jusqu'à l"Atlantique, en passant par le Saguenay. La croisière étant partie intégrante de l'évasion, la réalisation concrète de l'itinéraire aurait en quelque sorte constitué le Saint-Laurent lui-même en $\alpha$ attracteur $»$ et ce, depuis les Grands Lacs jusqu'à la baie des Ha! Ha! (Richelieu \& Ontario Navigation Co, 1896 : Canada Steamship Line Co. 1945). En 1894,

Lemprise de la $R$ \& O continua de s'affirmer sous la présidence du plus puissant homme d'affaines canadienfrançais de l'époque, Louis-Joseph Forget. Celui-ci accorda une place plus importante aux services aux passagers sur l'ensemble du réseau et notanment aux croisières sur le Saint-Lawent. En 1898 , il fait agrandir le vieil Hôtel Tadoussac et, I'an- 
nee suivante, il fait construire le premier Manoir Richelieu. Cette infrastructure hoteliere lui permit de concurrencer plus efficacement son grand rival, la St. Lawrence Steam Navigation Company de la famille Molson et de prendre le leadership du marché des croisières (Tittley, 1995: 13).

En cette dynamique géopolitique d'appropriation territoriale, pourrait-on trouver la clé d'interprétation de la confluence Saguenay-Saint-Laurent - non plus séparateur de rives mais a gradient $*$ régional fédérateur - telle que proposée par Lorraine Guay (2000) dans sa récente et monumentale thèsé sur le Saint-Laurent insulaire?

Et cette dynamique - faut-il le répéter? - aura été sous-jacente aux compétitions économiques ou encore « techniques s, entre le rail et l'eau par exemple. Elle a procédé de rivalités d'appropriations qui. sous ce rapport précis, ont confronté des bourgeoisies \& domestiques * et culmine dans la formation d'un monopole sur le Saint-Laurent : la Canadian Steamship Lines.

Serge Gagnon est professeur au Centre d'érudes collegiales en Charlevoix et chargé de cours au Département de géographie de l'UOAM.

\section{Note}

1 Cette aire touristique omprend Charlevoix. le Bas-Saguenay, la Côte-du-Sud et le Bas Saint-Laurent.

\section{Bibliographie}

Auger, C. (1993), a A la recherche du pittoresque dans l'Estries, Cap-awx-Diamans, 33, p. $59-62$.

Beaudet, Gérard, et Claude Lamothe (1996), a Du site au territoire : l'aménagement touristique du Québec w., Trames, 11, p. 63-69.

Bergeron. Claude (1989), Architectures du $X X^{e}$ siècle au Québèc, Montréal, Méridien. $271 \mathrm{p}$.

Bigsby, John-J. (1850), The Shoe and Canoe, or Pictures of Travels dans The Canadas. London, Chapman and Hall.
Blanchard, Raoul (1960), * Le tourisme * dans Le Canada francais, Province de Québec, Etude géographique, Montréal, Fayard, p. 235-245.

Brière, Roger (1967), * Les grands traits de l'évolution du tourisme au Québec s. Bulletin de l'association des géographes de l'Amérique française, 11 , p. 83-95.

Canada Steamship Line Co, (1945), The Sague= nay Trip, Montréal, La Patrie Printing.

De Blois-Martin, Charles (1997), * Tourisme et patrimoine: les rendez-vous manqués * Continulte, 76 , p. $37-40$.

Des Gagniers, Jean (1994), Charlevoix, pays enchante, Québec, Les Presses de l'Universite Laval, p. 362-363.

Dube, Philippe (1983), * Faire l'histoire du pays visité : Charlevoix *, Loisir es Société, 6 (1), P. 211-228.

Dubé, Philippe (1985), * Charlevoix: nos châteaux de la Loire $\%$, Continuite, p. 34-35.

Dube, Philippe (1986), Deux cents ans de villégiature dans Charlevoix : L'histoire d'un pays visité, Québec, Les Presses de l'Université Laval, 336 p.

Dubé, Philippe (1995), \& La villégiature dans Charlevoix : une tradition séculaire, un patrimoine encore vivant ${ }_{\text {, }}$,Eoros, 14 (2), p. 4-7. Gagnon, France (1992), \&u cheval au rail : l'évolution des circuits touristiques québécois au XIXe siècle ${ }^{2}$, dans Le pays laurentien au $X I X$ siecle. Cahier 1 , Universite Laval, Université du Québec à Montréal, Université du Québec à Trois-Rivières, p. 101-133.

Gagnon, Serge (1998), a De Lower St. Lawrence à Charlevoix : l'émergence d'un haut lieu de villégiature de la bourgeoisie marchande canadienne $\%$, Téoros, 17 (1), p. 15-22.

Gagnon, Serge (1996), L'émergence du tourismé au $X I X$ siècle : l'exemple de Charlevoix. Une analyse morphologique, dynamique et sémiotique, Université Laval, Département de Géographie, Mémoire de maîtrise.

Guay, Lorraine (2000), Le Sain-Laurent insulaire, Université Laval, Département de Gếographie, Thèse de doctorat.

Laberge, Alain (1993), Histoire de la Côte-duSrd, Collection Les régions du Québec, Québec, IQRC, 644 p.

Larocgue, Paul (1993), Parcours touristiques dans la region touristique du Bas-Saint-Laurent, Rimouski, GRIDEQ.

Lessard, Michel (1993), a La vogue des bateaux blancs s, Cap-aux-Diamants, 33, p. 50-52.

MacPherson Le Moine, James (1872), L'album du touriste: archeologie, histoire, litterature. sport, Québee, Augustin Côté et Cic.
Martin, Julie (1998), \& Kamouraska sur le quivive! $\%$, Continaile, 78, p. 30-31.

Normand, France (1997), Naviguer le SaintLaurent a la fin du XIX siecle. Une einde de la batellerie du port de Quebec, Collection Geographie historique, Québec, Les Presses de l'Université Laval, 283 p.

Paradis, Alexandre (1948), Kamouraska (16741948), Québec, $394 \mathrm{p}$.

Prévost, Robert (2000), Trois siecles de tourisme au Québec, Sillery, Septentrion, 365 p. Richelicu \& Ontario Navigation Co. (1896), From Niagara to the Sea: Descriptive of That Delightul Trip Down the River St. Lawrence and Up the World-Famed Saguenay, Montréal.

Ritchot, Gilles et al (1981), Enviromement de qualite et rente fonciere, Volume 2 de 3 dossiers, Québec, CRAD, Université Laval.

Saint-Amour, Jean-Pierre (1979), La villegiafure au Québec, problématique de l'anénagement du territoire, Hull, Edition Asticou. 178 p. Samson, Marcel (1987), * La villégiature, un lien historique $\times$, Téoros, 6 (2), p. 6-8.

Samson, Marcel (1988), « La route des villegiateurs. Dans sa quête d'un refuge estival, une aristocratie de vacanciers a tracé l'cs-

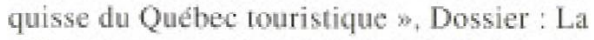
villégiature au Québec, Continuite, 40, p. 12 15 .

Stafford, Jean, et Marcel Samson (1986), « L'industric touristique québecoise ; entre le passé et l'avenir *, Les pratiques culturelles des québécois, Québec, IQRC : 291-317.

Tittley, Luc (1995), * Les croisières sur le SaintLaurent... un peu d'histoire *, Téoros, 14 (2), p. 12-14.

Vézina, Raymond (1977), * L'art documentaire au service des sciences humaines : le cas du comté de Charlevoix au Québec s, Cahiers de géographie de Quebec, 21 (53-54), p. 293-308.

Villeneuve, Lynda (1996), \& Mythe et vécu territorial : Charlevoix à travers l'art du paysage au XIX siècle $\mathrm{x}$, Cahiers de géographie du Quebec, 40 (111), p. 341-362.

Villeneuve, Lynda (1999), Paysage, mythe et territorialite : Charlevoix au XIX siecle, pour une nouvelle approche du paysage, Collection Géographie historique, Québec, Les Presses de l'Universite Laval. 